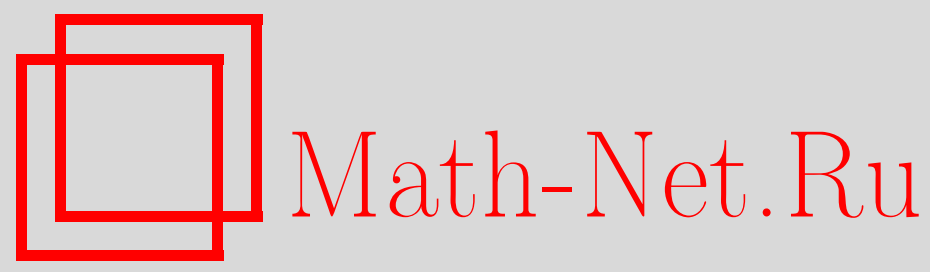

Г. Г. Ониани, О возможных значениях верхней и нижней производных относительно выпуклых дифференциальных базисов, Матем. заметки, 2004, том 76, выпуск 5, 762-775

DOI: https://doi.org/10.4213/mzm146

Использование Общероссийского математического портала Math-Net.Ru подразумевает, что вы прочитали и согласны с пользовательским соглашением http://www.mathnet.ru/rus/agreement

Параметры загрузки:

IP: 107.22 .136 .117

26 апреля 2023 г., 14:38:19

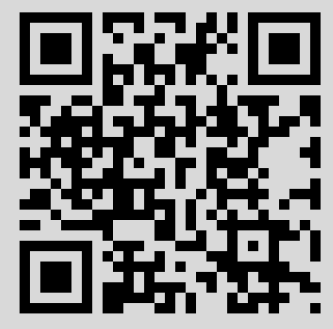




\section{О ВОЗМОЖНЫХ ЗНАЧЕНИЯХ ВЕРХНЕЙ И НИЖКЕЙ ПРОИЗВОДНЫХ ОТНОСИТЕЛЬНО ВЫПУКЛЫХ ДИФФЕРЕНЦИАЛЬНЫХ БАЗИСОВ}

\section{Г.Г. Ониани}

Доказано, что если выпуклый плотностный дифференциальный базис $B$ является центрированньм и инвариантньм относительно сдвига и гомотетии, то интегральные средние неотрицательной суммируемой функции, взятыепо $B$, могут расходиться ограниченно лишь на множестве меры нуль (тем самым, дано обобщение соответствующей теоремы Гусмана и Менаргеса), причем установлено, что инвариантность ни относительно сдвига, ни относительно гомотетии не является излишним условием.

Библиография: 14 названий.

Определения и обозначения. Отображение $B$, определенное на $\mathbb{R}^{n}$, называется дифференииальным базисом в $\mathbb{R}^{n}$, если для каждой $x \in \mathbb{R}^{n} B(x)$ является семейством открытых ограниченных множеств, содержащих точку $x$, таких, что существует последовательность $\left\{R_{k}\right\} \subset B(x) \mathrm{c} \operatorname{diam} R_{k} \rightarrow 0, k \rightarrow \infty$.

Для базиса $B$ через $\bar{B}$ будем обозначать семейство $\bigcup_{x \in \mathbb{R}^{n}} B(x)$.

Пусть $B$ - базис в $\mathbb{R}^{n}$. Для $f \in L_{\mathrm{loc}}\left(\mathbb{R}^{n}\right)$ и $x \in \mathbb{R}^{n}$ верхний и нижний предел интегральных средних $\frac{1}{|R|} \int_{R} f$, где $R$ - произвольное множество из $B(x)$ и $\operatorname{diam} R \rightarrow 0$, назьваются верхними и нижними производными интеграла от $f$ в точке $x$ и обозначаются через $\bar{D}_{B}\left(\int f, x\right)$ и $\underline{D}_{B}\left(\int f, x\right)$ соответственно. Если они равны, то их общее значение назьвают производным интеграла от $f$ в точке $x$ и обозначают через $D_{B}\left(\int f, x\right)$. В дальнейшем, ради краткости, интеграл в записях производных будем опускать.

Говорят, что базис $B$ дифферениирует $\int f$, если $\bar{D}_{B}(f, x)=\underline{D}_{B}(f, x)=f(x)$ для почти всех $x \in \mathbb{R}^{n}$.

Максимальный оператор $M_{B}$, соответствующий базису $B$, определяется равенством

$$
M_{B}(f)(x)=\sup _{R \in B(x)} \frac{1}{|R|} \int_{R}|f|, \quad f \in L_{\mathrm{loc}}\left(\mathbb{R}^{n}\right), \quad x \in \mathbb{R}^{n} ;
$$

обозначим также для $r>0$

$$
\begin{aligned}
& M_{B}^{(r)}(f)(x)=\sup _{R \in B(x), \operatorname{diam} R<r} \frac{1}{|R|} \int_{R}|f| \\
& \bar{M}_{B}^{(r)}(f)(x)=\sup _{R \in B(x), \operatorname{diam} R<r} \frac{1}{|R|} \int_{R} f .
\end{aligned}
$$


Базис $B$ назовем

- образованным множествами из некоторого класса $\Delta$, если $\bar{B} \subset \Delta$;

- выпуклым, если он образован из выпуклых множеств;

- плотностным, если для каждого измеримого множества $E$ базис $B$ дифференцирует $\int \chi_{E}$;

- инвариантным относительно сдвига, если $B(x)=\{R+x: R \in B(O)\}$ для каждой $x \in \mathbb{R}^{n}$, где $O$ - начало координат в $\mathbb{R}^{n}$;

- инвариантным относительно гомотетии, если для каждых $x \in \mathbb{R}^{n}, R \in B(x)$ и гомотетии $H$ с центром в $x$ имеем $H(R) \in B(x)$;

- буземан-феллеровским, если для каждого $R \in \bar{B}$ имеем $R \in B(x)$ для каждого $x \in R$;

- измеримым, если для каждых $f \in L\left(\mathbb{R}^{n}\right)$ и $r>0$ функция $\bar{M}_{B}^{(r)}(f)$ измерима.

Заметим, что

1) если $B$ - плотностный базис, то $B$ дифферениирует интеграл от каждой измеримой ограниченной функиии (см., например, [1, гл. III, § 2]);

2) измеримость базиса $B$ влечет за собой измеримость функиий $\bar{D}_{B}(f, \cdot)$, $\underline{D}_{B}(f, \cdot), M_{B}(f) u M_{B}^{(r)}(f)$ для каждых $f \in L\left(\mathbb{R}^{n}\right)$ u $r>0 ;$

3) если базис В инвариантен относительно сдвига или является буземан-феллеровским, то $B$ измерим;

4) если базис $B$ является буземан-феллеровским и инвариантен относительно гомотетии, то В инвариантен и относительно сдвига.

Утверждения 2)-4) проверяются непосредственно.

Через $\mathbb{I}\left(\mathbb{R}^{n}\right)$ обозначим базис, для которого $\mathbb{I}\left(\mathbb{R}^{n}\right)(x), x \in \mathbb{R}^{n}$, состоит из всех $n$-мерных интервалов, содержащих $x$. Дифференцирование относительно $\mathbb{I}\left(\mathbb{R}^{n}\right)$ принято назьвать сильным дифферениированием.

Прямоугольным репером в $\mathbb{R}^{n}$ назовем множество, состоящее из $n$ взаимоортогональных прямых, проходящих через начало координат. Пусть $E$ - некоторое семейство прямоугольных реперов в $\mathbb{R}^{n}$. Через $\mathbb{I}_{E}\left(\mathbb{R}^{n}\right)$ обозначим базис, для которого $\mathbb{I}_{E}\left(\underline{\mathrm{R}}^{n}\right)(x)$, $x \in \mathbb{R}^{n}$, состоит из всех $n$-мерных прямоугольных параллелепипедов, содержащих $x$, ребра которых параллельны соответствуюшим прямым некоторого репера из $E$.

Базисы $B_{1}$ и $B_{2}$ назовем эквивалентными (положительно әквивалентными), если для каждой $f \in L\left(\mathbb{R}^{n}\right)\left(f \in L\left(\mathbb{R}^{n}\right), f \geqslant 0\right) \underline{D}_{B_{1}}(f, x)=\underline{D}_{B_{2}}(f, x)$ и $\bar{D}_{B_{1}}(f, x)=$ $\bar{D}_{B_{2}}(f, x)$ для почти всех $x \in \mathbb{R}^{n}$.

Пусть $E$ - вьпуклое ограниченное открытое множество и $x \in E$. Для единичного вектора е рассмотрим числа $\sup \{t>0: x+t e \in E\}, \sup \{t>0: x-t e \in E\}$ и отношение минимального к максимальному среди них обозначим через $c_{e}(x, E) ; c(x, E)$ определим как $\inf _{e} c_{e}(x, E)$, где инфимум берется по всем единичным векторам; $c(x, E)$ выражает “коэффициент центрирования" множества $E$ в точке $x$.

Выпукльй базис $B$ назовем иентрированным, если существует $\varepsilon>0$ такое, что для каждых $x \in \mathbb{R}^{n}$ и $E \in B(x)$ существует сдвиг $T$, для которого $T(E) \in B(x)$ и $c(x, T(E)) \geqslant \varepsilon$.

Нетрудно проверить, что каждый выпукльй буземан-феллеровский базис является иентрированным.

Через $\mathbb{G}^{n}$ будем обозначать единичньй куб в $\mathbb{R}^{n}$, т.е. $\mathbb{G}^{n}=[0,1]^{n}$. 
Результаты. Отвечая на вопрос, поставленньй Саксом [2] Безикович [3] (см. также $[1$, гл. IV,$\S 3])$ доказал, что сильные интегральные средние суммируемой на плоскости функиии могут расходиться ограниченно только на множестве мерь нуль; точнее, для каждой $f \in L\left(\mathbb{R}^{2}\right)$ оба множества

$$
\left\{-\infty<\underline{D}_{\mathbb{I}\left(\mathbb{R}^{2}\right)}(f, \cdot)<f\right\}, \quad\left\{f<\bar{D}_{\mathbb{I}\left(\mathbb{R}^{2}\right)}(f, \cdot)<\infty\right\}
$$

имеют меру нуль. Многомерный аналог этого результата был установлен Вардом [4] (см. также [5, гл. IV, § 11]).

Целью настоящей работы является изучение следующей задачи, поставленной Гусманом [6, с. 389]: на какие базисы можнно распространить теорему Безиковича?

В дальнейшем будем говорить, что базис $B$ в $\mathbb{R}^{n}$ имеет свойство Безиковича (слабое свойство Безиковича), если множества $\left\{-\infty<\underline{D}_{B}(f, \cdot)<f\right\},\left\{f<\bar{D}_{B}(f, \cdot)<\infty\right\}$ имеют меру нуль для каждой $f \in L\left(\mathbb{R}^{n}\right)\left(f \in L\left(\mathbb{R}^{n}\right), f \geqslant 0\right)$.

Класс известных базисов, имеющих свойство Безиковича, небогат. В частности, кроме $\mathbb{I}\left(\mathbb{R}^{n}\right)$, свойством Безиковича обладает и любой плотностный базис $\mathbb{I}_{E}\left(\mathbb{R}^{n}\right)$. Это можно доказать, пользуясь многомерным аналогом леммы Рисса "о восходящем солнце", установленной в [7], и рассуждая по схеме из [3]. Здесь же заметим, что Зерекидзе [8], [9] указал класс базисов, образованных из $n$-мерных интервалов, которые эквивалентны $\mathbb{I}\left(\mathbb{R}^{n}\right)$ и, следовательно, имеют свойство Безиковича.

Что же касается базисов со слабым свойством Безиковича, для них известна следующая довольно общая

Теорема (Гусман-Менаргес [1, гл. IV, §3]). Если выпуклый плотностный базис $B$ в $\mathbb{R}^{n}$, образованный из иентрально-симметричных мнохсеств, является буземан-феллеровским и инвариантным относительно гомотетии, то $B$ имеет слабое свойство Безиковича.

Усилением этого результата является

ТЕОРемА 1. Если выпуклый плотностный базис $B$ в $\mathbb{R}^{n}$ одновременно являетcя:

1) инвариантным относительно сдвига,

2) инвариантным относительно гомотетии,

3) ченрированным,

то В имеет слабое свойство Безиковича.

Нижеприведенные утверждения показьвают, в частности, что условия 1) и 2) в теореме 1 не являются излишними.

ТЕОРема 2. Существует инвариантный относительно гомотетии центрированный базис $B$, образованный из двумерных интервалов, для которого найдется функиия $f \in L\left(\mathbb{G}^{2}\right), f \geqslant 0$, такая, что

$$
f(x)<\bar{D}_{B}(f, x)<\infty \quad \text { почти всюдуна } \mathbb{G}^{2} .
$$

ТЕОРема 3 [10]. Существует инвариантный относительно сдвига буземан-феллеровский базис $B$, образованный из двумерных интервалов, для которого найдется функиия $f \in L\left(\mathbb{G}^{2}\right), f \geqslant 0$, такая, что

$$
f(x)<\bar{D}_{B}(f, x)<\infty \quad \text { почти всюдуна } \mathbb{G}^{2} .
$$


ЗАмечАниЕ 1 . Теорема 1 в случае требования буземан-феллеровости вместо условия центрированности, а теорема 2 без требования центрированности были установлены в [11].

ЗАмЕчанИЕ 2. Нам не известен ответ на вопрос: является ли условие иентрированности в теореме 1 излишним, т.е. имеет ли кажддй выпуклый плотностный и инвариантный относительно сдвига и гомотетии базис свойство Безиковича.

Доказательства. Пусть $B$ - вьпукльй базис в $\mathbb{R}^{n}$ такой, что множества из $\bar{B}$ попарно сравнимы, т.е. для каждых $E_{1}, E_{2} \in \bar{B}$ существует сдвиг одного из них, который помешает его внутрь другого. Тогда (см. [1, гл. I, $\S 3$, замечание (1)]) для $B$ справедлива теорема о покрытии типа теоремы Витали, откуда с помощью стандартной техники (см., например, $\left[1\right.$, гл. I, $\S 3$, замечание (9)]) выводится, что $B$ дифференцирует $L\left(\mathbb{R}^{n}\right)$. Из этого замечания непосредственно вытекает

ЛЕмма 1. Пусть В - инвариантный относительно сдвига выпукльй базисв в $\mathbb{R}^{n}$. Тогда найдется его подбазис $B^{\prime}$, дифферениирующий $L\left(\mathbb{R}^{n}\right), u$, следовательно, для каждой $f \in L\left(\mathbb{R}^{n}\right)$

$$
\underline{D}_{B}(f, x) \leqslant f(x) \leqslant \bar{D}_{B}(f, x) \quad \text { почти всюду на } \mathbb{R}^{n}
$$

Действительно, $B^{\prime}$ можно опеределить следующим образом: $B^{\prime}(x)=\left\{x+R_{k}: k \in \mathbb{N}\right\}$, $x \in \mathbb{R}^{n}$, где $\left\{R_{k}\right\} \subset B(0), \operatorname{diam} R_{k} \rightarrow 0, k \rightarrow \infty$ и $R_{1} \supset R_{2} \supset \cdots$

Лемма 2. Пусть $B-$ плотностный базис в $\mathbb{R}^{n}$. Тогда для каждой $f \in L\left(\mathbb{R}^{n}\right)$, $f \geqslant 0$, имеем $\left|\left\{\underline{D}_{B}(f, \cdot)<f\right\}\right|=0$.

ДоказАТЕЛЬСтво. Пусть $f \in L\left(\mathbb{R}^{n}\right)$ и $f \geqslant 0$. Рассмотрим произвольное $\varepsilon>0$, а $\alpha>0$ подберем так, чтобы $\int_{\{f>\alpha\}} f<\varepsilon^{2}$. Обозначим $f_{*}=f \chi_{\{f \leqslant \alpha\}}, f^{*}=f \chi_{\{f>\alpha\}}$. Ясно, что $\left\{\underline{D}_{B}\left(f^{*}, \cdot\right)<f^{*}-\varepsilon\right\} \subset\left\{f^{*}>\varepsilon\right\}$, откуда

$$
\left|\left\{\underline{D}_{B}\left(f^{*}, \cdot\right)<f^{*}-\varepsilon\right\}\right| \leqslant\left|\left\{f^{*}>\varepsilon\right\}\right| \leqslant \frac{1}{\varepsilon} \int_{\mathbb{R}^{n}} f^{*}<\varepsilon .
$$

Так как плотностные базисы дифференцируют интегралы от ограниченных измеримых функций, то $B$ дифференцирует $\int f_{*}$. Следовательно, с учетом (1) легко видеть, что

$$
\left|\left\{\underline{D}_{B}(f, \cdot)<f-\varepsilon\right\}\right|=\left|\left\{\underline{D}_{B}\left(f^{*}, \cdot\right)<f^{*}-\varepsilon\right\}\right|<\varepsilon .
$$

Откуда в силу произвольности $\varepsilon>0$ заключаем справедливость леммы. Лемма 2 доказана.

Ниже, ради краткости, вместо прямоугольного параллелепипеда будем писать просто прямоугольник.

Если $E$ - центрально-симметричное множество в $\mathbb{R}^{n}$ с центром в $x$ и $\alpha>0$, то через $\alpha E$ будем обозначать раздутие $E$ в $\alpha$ раз, т.е. множество $\{x+\alpha(t-x): t \in E\}$. 
Лемма 3. Пусть $E$ - открытое ограниченное и выпуклое множество в $\mathbb{R}^{n}$. Тогда

а) существует прямоугольник $R$ такой, что $R \subset E \subset n^{2} R$;

b) для каждой точки $x \in E$ существует прямоугольник $R_{x}$, гомотетичный $R$ и с иентром в $x$, такой, что

$$
R_{x} \subset E \subset \frac{4 n^{2}}{c(x, E)} R_{x}
$$

ДокАЗАТЕЛЬСтво. а) Известно, что (см., например, [1, гл. VI, § 2]) найдется открытый эллипсоид $T$ такой, что $T \subset E \subset n T$. Далее, выбирая в качестве $R$ прямоугольник $\frac{1}{n} R^{\prime}$, где $R^{\prime}$ - минимальньй среди открытых прямоугольников, содержащих $T$ и имеющих ребра, параллельные главным осям $T$, легко видеть, что $R \subset T \subset n R$, а следовательно, $R \subset E \subset n^{2} R$.

b) В силу уже доказанного пункта а) найдется прямоугольник $R$ такой, что

$$
R \subset E \subset n^{2} R
$$

Пусть $y$-центр $R$ и $z=x-c(x, E)(y-x)$. Из вьпуклости $E$ и определения $c(x, E)$ следует, что $z \in E$. Рассмотрим гомотетию $H$ с центром в $z$ и с коэффициентом $c(x, E) /(1+$ $c(x, E))$. Так как $H(y)=x$, центром прямоугольника $H(R)$ является $x$. В силу вьпуклости $E$ имеем

$$
H(R) \subset E \text {. }
$$

Из (2), (3) и определения $H$ легко видеть, что

$$
E \subset n^{2} R \subset n^{2}\left[2 \frac{1+c(x, E)}{c(x, E)} H(R)\right] \subset \frac{4 n^{2}}{c(x, E)} H(R) .
$$

Таким образом, прямоугольник $R_{x}=H(R)$ удовлетворяет всем нужным условиям. Лемма доказана.

Нам понадобится следующая теорема, по существу принадлежащая Буземану и Феллеру [12] (см. также [1, гл. III, $\S 1]$ ).

ТЕОремА. Пусть В является измеримым базисом. Два следующих свойства әквивалентны:

a) В есть плотностный базис;

b) для каждого $\lambda, 0<\lambda<1$, для каждой последовательности ограниченных измеримых мнохеств $\left\{A_{k}\right\}$ таких, что $A_{1} \supset A_{2} \supset \cdots u\left|A_{k}\right| \rightarrow 0, k \rightarrow \infty$, и для каждой последовательности чисел $\left\{r_{k}\right\}$ таких, что $r_{1}>r_{2}>\cdots u$ $r_{k} \rightarrow 0, k \rightarrow \infty$, имеем $\left|\left\{M_{B}^{\left(r_{k}\right)}\left(\chi_{A_{k}}\right)>\lambda\right\}\right| \rightarrow 0, k \rightarrow \infty$.

Заметим, что это утверждение в [1] доказано при предположении буземан-феллеровости базиса $B$, однако, как видно из доказательства, такое требование нужно только ради обеспечения измеримости $\bar{D}_{B}(f, \cdot), \underline{D}_{B}(f, \cdot)$ и $M_{B}^{(r)}(f)$. Так что утверждение справедливо и для любых измеримых базисов. 
Пусть $B$ - базис со свойствами 1)-3) из теоремы 1 и пусть для каждого $E \in B(O)$ с $\operatorname{diam} E=1 R_{E}$ - прямоугольник такой, что $R_{E} \subset E \subset n^{2} R_{E}$ (такой прямоугольник существует в силу леммы 3$)$. Рассмотрим семейство $\Delta$ всех множеств вида $(T \circ H)\left(R_{E}\right)$, где $T$ - некоторый сдвиг, $H$-некоторая гомотетия с центром в $O, E \in B(O)$ и $\operatorname{diam} E=1$. Через $B^{*}$ обозначим базис, определенньй следуюшим образом:

$$
B^{*}(x)=\{R: R \in \Delta, R \ni x\}, \quad x \in \mathbb{R}^{n} .
$$

Для $B$ и $B^{*}$ справедлива

Лемма 4. а) $B^{*}$ является буземан-феллеровским плотностным базисом, инвариантным относительно сдвига и гомотетии.

b) Найдется $\varepsilon>0$ такое, что для каждого $f \in L\left(\mathbb{R}^{n}\right), f \geqslant 0$,

$$
\bar{D}_{B}(f, \cdot) \leqslant\left(\frac{12 n^{2}}{\varepsilon}\right)^{n} \bar{D}_{B^{*}}(f, \cdot) \quad u \quad \bar{D}_{B^{*}}(f, \cdot) \leqslant\left(\frac{12 n^{2}}{\varepsilon}\right)^{n} \bar{D}_{B}(f, \cdot) .
$$

ДокАЗАТЕЛЬСтво. Очевидно, $B^{*}$ является буземан-феллеровским базисом, инвариантньм относительно сдвига и гомотетии.

Из свойств $B$ и определения $B^{*}$ с учетом леммы 3 нетрудно проверить, что найдется $\varepsilon>0$ такое, что

i) для каждого $E \in B(x)$ найдется $R \in B^{*}(x)$ и сдвиг $T$ такие, что $T(R) \subset E \subset$ $\left(12 n^{2} / \varepsilon\right) R$

ii) для каждого $R \in B^{*}(x)$ найдется $E \in B(x)$ и сдвиг $T$ такие, что $R \subset 3 E$ и $E \subset\left(4 n^{2} / \varepsilon\right) T(R)$.

Из і) и іi) заключаем, что для каждых $f \in L\left(\mathbb{R}^{n}\right), r>0$ и $x \in \mathbb{R}^{n}$

$$
\begin{aligned}
& M_{B}^{(r)}(f)(x) \leqslant\left(\frac{12 n^{2}}{\varepsilon}\right)^{n} M_{B^{*}}^{\left(\left(12 n^{2} / \varepsilon\right) r\right)}(f)(x), \\
& M_{B^{*}}^{(r)}(f)(x) \leqslant\left(\frac{12 n^{2}}{\varepsilon}\right)^{n} M_{B}^{\left(\left(12 n^{2} / \varepsilon\right) r\right)}(f)(x) .
\end{aligned}
$$

Из (4) и (5) вытекают нужные нам оценки для верхних производньгх.

И, наконец, из (5) в силу вьшеотмеченной теоремы Буземана и Феллера заключаем, что $B^{*}$ является плотностным базисом. Лемма доказана.

ДоКАЗАТЕЛЬСТво ТЕОРЕмЫ 1 . Пусть базис $B^{*}$ определен так же, как в лемме 4. Очевидно, $B^{*}$ удовлетворяет всем условиям теоремы Гусмана и Менаргеса, поэтому $B^{*}$ имеет слабое свойство Безиковича.

Рассмотрим произвольную функцию $f \in L\left(\mathbb{R}^{n}\right), f \geqslant 0$. В силу лемм 1 и 2

$$
\underline{D}_{B}(f, x)=\underline{D}_{B^{*}}(f, x)=f(x) \quad \text { почти всюду на } \mathbb{R}^{n} .
$$

Обозначим $A_{1}=\left\{x: \bar{D}_{B^{*}}(f, x)=\infty\right\}, A_{2}=\left\{x: \bar{D}_{B^{*}}(f, x)=f(x)\right\}$. Из леммы 1 и из того, что $B^{*}$ имеет слабое свойство Безиковича, заключаем

$$
\left|\mathbb{R}^{n} \backslash\left(A_{1} \cup A_{2}\right)\right|=0 .
$$


На основании леммы 4 пишем

$$
\bar{D}_{B}(f, x)=\bar{D}_{B^{*}}(f, x)=\infty \quad \text { при } \quad x \in A_{1} .
$$

Теперь покажем, что

$$
\bar{D}_{B}(f, x)=\bar{D}_{B^{*}}(f, x)=f(x) \quad \text { почти всюду на } A_{2} \text {. }
$$

Рассмотрим произвольное $\delta>0$. Число $\alpha$ подберем так, чтобы

$$
\frac{1}{\delta}\left(\frac{12 n^{2}}{\varepsilon}\right)^{n} \int_{\{f>\alpha\}} f<\delta,
$$

где $\varepsilon>0$ - число из леммы 4. Обозначим $f^{*}=f \chi_{\{f>\alpha\}}, f_{*}=f \chi_{\{f \leqslant \alpha\}}$. Так как $B^{*}$ является плотностньп, то $B^{*}$ дифференцирует $\int f_{*}$, откуда с учетом леммы 4 легко видеть, что

$$
\begin{aligned}
\left|\left\{x \in A_{2}: \delta<\bar{D}_{B}\left(f^{*}, x\right)\right\}\right| & \leqslant\left|\left\{x \in A_{2}: \delta<\left(\frac{12 n^{2}}{\varepsilon}\right)^{n} D_{B^{*}}\left(f^{*}, x\right)\right\}\right| \\
& =\left|\left\{x \in A_{2}: \delta<\left(\frac{12 n^{2}}{\varepsilon}\right)^{n} f^{*}(x)\right\}\right| \\
& \leqslant \frac{1}{\delta}\left(\frac{12 n^{2}}{\varepsilon}\right)^{n} \int_{\mathbb{R}^{n}} f^{*}<\delta .
\end{aligned}
$$

Базис $B$ является плотностным, поэтому $B$ дифференцирует $\int f_{*}$. Следовательно, из (10) пишем

$$
\begin{aligned}
\mid\{x & \left.\in A_{2}: f(x)+\delta<\bar{D}_{B}(f, x)\right\} \mid \\
& \leqslant\left|\left\{x \in A_{2}: f^{*}(x)+\varepsilon<\bar{D}_{B}\left(f^{*}, x\right)\right\}\right|+\left|\left\{x \in A_{2}: f_{*}(x)<\bar{D}_{B}\left(f_{*}, x\right)\right\}\right|<\delta+0=\delta .
\end{aligned}
$$

Этим, очевидно, (9) доказано.

Из (6)-(9) заключаем справедливость теоремы 1.

ЗАмЕчАнИЕ 3. При доказательстве теоремы 1 мы дополнительно установили, что существует инвариантньй относительно сдвига и гомотетии буземан-феллеровский базис $B^{*}$, образованньй из $n$-мерных прямоугольников, которьй положительно эквивалентен $B$.

ЗАмЕЧАНИЕ 4. Можно доказать следующую теорему, которая выражает общий принцип, которым мы пользовались для доказательства теоремы 1.

Теорема. Пусть $B$ - плотностный базис, В $^{*}$ имеет слабое свойство Безиковича и для каждой $f \in L\left(\mathbb{R}^{n}\right)$

$$
\underline{D}_{B}(f, x) \leqslant f(x) \leqslant \bar{D}_{B}(f, x) \quad \text { почти всюду на } \mathbb{R}^{n} \text {. }
$$

Если, кроме того, для каждой $f \in L\left(\mathbb{R}^{n}\right), f \geqslant 0$, найдется $c=c(f) \geqslant 1$ такое, что для кажсдого $\lambda>0$

$$
\left|\left\{\bar{D}_{B}(f, \cdot)>\lambda\right\}\right| \leqslant c\left|\left\{\bar{D}_{B^{*}}(f, \cdot)>\frac{\lambda}{c}\right\}\right|, \quad\left|\left\{\bar{D}_{B^{*}}(f, \cdot)>\lambda\right\}\right| \leqslant c\left|\left\{\bar{D}_{B}(f, \cdot)>\frac{\lambda}{c}\right\}\right|,
$$


то В положительно әквивалентен $B^{*}$ и, следовательно, имеет слабое свойство Безиковича.

Приведенные ниже леммы нам понадобятся для доказательства теоремы 2.

Всюду ниже мы рассматриваем двумерные интервалы.

Пусть для интервала $I$ :

- $\alpha_{I}$ - длина большей стороны $I, \beta_{I}$ - длина меньшей стороны $I$ и $r(I)$ - коэффициент регулярности $I$, т.е. число $\alpha_{I} / \beta_{I}$;

- $\Lambda(I, h), h>1,-$ семейство всех интервалов $J$ со свойствами: $J \supset I,|J|=h|I|$ и одна из вершин $J$ совпадает с одной из вершин $I$;

$$
R(I, h)=\left(3 \mathrm{pr}_{1} I \times(2 h-1) \mathrm{pr}_{2} I\right) \cup\left((2 h-1) \mathrm{pr}_{1} I \times 3 \mathrm{pr}_{2} I\right), \quad h>1
$$

где $\mathrm{pr}_{1}$ и $\mathrm{pr}_{2}-$ проекции в $\mathbb{R}^{2}$.

Ниже вместо $\mathbb{I}\left(\mathbb{R}^{2}\right)$ будем писать просто $\mathbb{I}$.

Следующее утверждение проверяется непосредственно.

Лемма 5. Пусть $I=\left[\alpha_{1}, \alpha_{1}\right] \times\left[-\alpha_{2}, \alpha_{2}\right]$, əде $\alpha_{1}, \alpha_{2}>0 u h>1$. Тогда

1) $\left\{M_{\mathbb{I}}\left(h \chi_{I}\right)>1\right\}=\left\{x \in \mathbb{R}^{2}:\left|x^{1}\right|<(2 h-1) \alpha_{1},\left|x^{2}\right|<(2 h-1) \alpha_{2},\left(\left|x^{1}\right|+\alpha_{1}\right) \times\right.$ $\left.\left(\left|x^{2}\right|+\alpha_{2}\right)<4 h \alpha_{1} \alpha_{2}\right\}$

2) $\left\{M_{\mathbb{I}}\left(h \chi_{I}\right)>1\right\} \subset(2 h-1) I$;

3) $\left|\left\{M_{\mathbb{I}}\left(h \chi_{I}\right)>1\right\}\right|=(4 h \ln h+1)|I|$.

Учитывая лемму 5, нетрудно убедиться, что справедлива

Лемма 6. Пусть $I$ - интервал $и$ - $>$ 1. Тогда

$$
\left\{M_{\mathbb{I}}\left(h \chi_{I}\right)>1\right\}=\bigcup_{J \in \Lambda(I, h)} J .
$$

Лемма 7. Пусть $I-$ интервал $u h>1$. Тогда для каждого $J \in \Lambda(I, h)$

$$
\frac{r(I)}{h} \leqslant r(J) \leqslant h r(I)
$$

ДоказАТЕЛЬСтво. Так как $I \subset J$ и $|J|=h|I|$, то $\beta_{J} \geqslant \beta_{I}$ и $\alpha_{J} \geqslant \alpha_{I}, r(J) \beta_{J}^{2}=$ $h r(I) \beta_{I}^{2}$ и $\alpha_{J}^{2} / r(J)=h \alpha_{I}^{2} / r(I)$. Откуда получаем, что $r(J) \leqslant h r(I)$ и $r(J) \geqslant r(I) / h$.

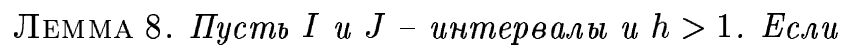

$$
r(J) \notin\left[\frac{r(I)}{h}, h r(I)\right] \quad u \quad \frac{h|J \cap I|}{|J|}>1,
$$

mo $J \subset R(I, h)$. 
ДоказАтельство. Очевидно, $|J|<h|I|$. Таким образом, $\alpha_{J}^{2} / r(J)<h \alpha_{I}^{2} / r(I)$ и $r(J) \beta_{J}^{2}<h r(I) \beta_{I}^{2}$, откуда в силу того, что $r(J) \notin[r(I) / h, h r(I)]$, получаем

$$
\alpha_{J}<\alpha_{I} \quad \text { или } \quad \beta_{J}<\beta_{I},
$$

откуда, в свою очередь, с учетом того, что $J \cap I \neq \varnothing$ и $\left\{M_{\mathbb{I}}\left(h \chi_{I}\right)>1\right\} \subset(2 h-1) I$ (см. лемму 5), заключаем $J \subset R(I, h)$.

Лемма 9. Пусть $I$ - интервал, $h>1$ и пусть $J \in \Lambda(I, h), x \in J u x \notin 3 I$. Тогда для каждой гомотетии $H$ с иентром в $x$ справедливо неравенство

$$
\frac{h|H(J) \cap I|}{|H(J)|} \leqslant 4 .
$$

ДоКАЗАТЕЛЬСТВО. Пусть $\alpha-$ коэффициент гомотетии $H$.

1. Если $\alpha \geqslant 1$, то $|H(J)| \geqslant|J|=h|I| ;$ следовательно,

$$
\frac{h|H(J) \cap I|}{|H(J)|} \leqslant 1 .
$$

2. Если $1 / 2 \leqslant \alpha<1$, то $|H(J)| \geqslant|J| / 4=h|I| / 4 ;$ следовательно,

$$
\frac{h|H(J) \cap I|}{|H(J)|} \leqslant 4 .
$$

3. Если же $\alpha<1 / 2$, то легко видеть, что $H(J) \cap I=\varnothing$, и, следовательно,

$$
\frac{h|H(J) \cap I|}{|H(J)|}=0 .
$$

Через $\bar{L}\left(\mathbb{R}^{n}\right)$ обозначим класс всех функций $f \in L\left(\mathbb{R}^{n}\right)$, для каждой из которых при любом $\varepsilon>0$ найдется непрерывная на $\mathbb{R}^{n}$ функция $g \in L\left(\mathbb{R}^{n}\right)$ такая, что $|g(x)| \leqslant|f(x)|$ п.в. на $\mathbb{R}^{n}$ и $\|f-g\|_{1}<\varepsilon$.

Следующее утверждение является частным случаем одной леммы из [13] (см. также $[11$, с. 52$])$.

ЛЕмма 10. Пусть $f_{k} \in \bar{L}\left(\mathbb{R}^{n}\right), f_{k} \geqslant 0, k \in \mathbb{N}, \lambda>0$ u nyсmь npu $k, m \in \mathbb{N} u$ $k \neq m$ выполнены условия

$$
\begin{gathered}
\operatorname{supp} f_{k} \cap \operatorname{supp} f_{m}=\varnothing, \quad \operatorname{supp} f_{k} \cap\left\{M_{\mathbb{I}}\left(f_{m}\right)>\lambda\right\}=\varnothing \\
\left\{M_{\mathbb{I}}\left(f_{k}\right)>\lambda\right\} \cap\left\{M_{\mathbb{I}}\left(f_{m}\right)>\lambda\right\}=\varnothing .
\end{gathered}
$$

Тогда

$$
\left\{M_{\mathbb{I}}\left(\sum_{k=1}^{\infty} f_{k}\right)>\lambda\right\}=\bigcup_{k=1}^{\infty}\left\{M_{\mathbb{I}}\left(f_{k}\right)>\lambda\right\} .
$$


ДОКАЗАТЕЛЬСТВО ТЕОРЕМЫ 2. Пусть $\left\{h_{k}\right\}$ - последовательность со свойствами

$$
1<h_{1}<h_{2}<\cdots, \quad \sum_{k=1}^{\infty} \frac{1}{h_{k}}<\infty \quad \text { и } \quad \sum_{k=1}^{\infty} \frac{\ln h_{k}}{h_{k}}=\infty .
$$

Рассмотрим также возрастаюшие последовательности натуральных чисел $\left\{n_{k}\right\}$ и $\left\{m_{k}\right\}$ такие, что

$$
\begin{aligned}
& n_{k}<m_{k}, \\
& \frac{1}{2^{n_{k+1}}}<\frac{1}{h_{k} 2^{m_{k}+1}}, \\
& 2^{m_{k+1}-n_{k+1}}>h_{k+1} h_{k} 2^{m_{k}-n_{k}} .
\end{aligned}
$$

Для каждого $k \in \mathbb{N}$ разобъем $\mathbb{G}^{2}$ на $q_{k}=2^{n_{k}+m_{k}}$ конгруентных интервалов $Q_{k, q}$, $q \in \overline{1, q_{k}}$, длины горизонтальных сторон которых равны $1 / 2^{n_{k}}$, а длины вертикальных сторон $-1 / 2^{m_{k}}$. При $k \in \mathbb{N}, q \in \overline{1, q_{k}}$ обозначим

$$
I_{k, q}=\frac{1}{2 h_{k}} Q_{k, q}, \quad S_{k, q}=\left\{M_{\mathbb{I}}\left(h \chi_{I_{k, q}}\right)>1\right\} .
$$

Согласно лемме 6

$$
S_{k, q}=\bigcup_{J \in \Lambda\left(I_{k, q}, h_{k}\right)} J
$$

Положим

$$
\widetilde{S}_{k, q}=\bigcup_{J \in \Lambda\left(I_{k, q}, h_{k}\right)} \frac{1}{2} J
$$

Если $\left\{I_{t}\right\}_{t \in \Delta}-$ произвольное семейство $n$-мерных интервалов и $\alpha \geqslant 1$, то, учитывая очевидное включение

$$
\bigcup_{t \in \Delta} \alpha I_{t} \subset\left\{M_{\mathbb{I}}\left(\chi_{E}\right)>\frac{1}{\alpha}\right\}
$$

где $E=\bigcup_{t \in \Delta} I_{t}$, из известного неравенства Йессена-Марцинкевича-Зигмунда [14] (см. также $[1$, гл. II, $\S 3$, замечание (3)]) пишем

$$
\left|\bigcup_{t \in \Delta} \alpha I_{t}\right| \leqslant c(\alpha)\left|\bigcup_{t \in \Delta} I_{t}\right|
$$

где $c(\alpha)$ зависит от $n$ и $\alpha$. С учетом этого факта заключаем, что $\left|\widetilde{S}_{k, q}\right| \geqslant\left|S_{k, q}\right| / c(\alpha)$.

В силу леммы 5 имеем $S_{k, q} \subset\left(2 h_{k}-1\right) I_{k, q} \subset Q_{k, q},\left|S_{k, q}\right|>h_{k} \ln h_{k}\left|I_{k, q}\right|$. Следовательно, при $k \in \mathbb{N}$ пишем

$$
\begin{aligned}
\left|\bigcup_{q=1}^{q_{k}} \widetilde{S}_{k, q}\right| & =\sum_{q=1}^{q_{k}}\left|\widetilde{S}_{k, q}\right| \geqslant \sum_{q=1}^{q_{k}} \frac{1}{c(\alpha)}\left|S_{k, q}\right|>\sum_{q=1}^{q_{k}} \frac{1}{c(\alpha)} h_{k} \ln h_{k}\left|I_{k, q}\right| \\
& =\sum_{q=1}^{q_{k}} \frac{1}{c(\alpha)} h_{k} \ln h_{k} \frac{\left|Q_{k, q}\right|}{4 h_{k}^{2}}=\frac{\ln h_{k}}{4 c(\alpha) h_{k}} .
\end{aligned}
$$


Для каждого $k \in \mathbb{N}$ рассмотрим те интервалы $Q_{k+1, q}, q \in \overline{1, q_{k+1}}$, которые содержатся в $\bigcup_{q=1}^{q_{k}} \widetilde{S}_{k, q}$ и их объединение обозначим через $O_{k}$. Легко видеть, что если $\left\{n_{k}\right\}$ и $\left\{m_{k}\right\}$ достаточно быстро стремятся к $\infty$, то

$$
\left|O_{k}\right| \geqslant \frac{1}{2}\left|\bigcup_{q=1}^{q_{k}} \widetilde{S}_{k, q}\right|
$$

Будем считать, что $\left\{n_{k}\right\}$ и $\left\{m_{k}\right\}$ подобраны так, что выполняется (16). Легко убедиться, что $\left\{O_{k}\right\}$ является последовательностью независимых множеств. Следовательно, из $(11),(15)$ и $(16)$ в силу леммы Бореля-Кантелли заключаем: $\varlimsup_{k \rightarrow \infty} O_{k}$ имеет полную меру в $\mathbb{G}^{2}$. Тем более

$$
\varlimsup_{k \rightarrow \infty} \bigcup_{q=1}^{q_{k}} \widetilde{S}_{k, q} \quad \text { имеет полную меру в } \mathbb{G}^{2} .
$$

Пусть для $x \in \widetilde{S}_{k, q} \Delta_{k}(x)=\left\{I \in \Lambda\left(I_{k, q}, h_{k}\right): I \ni x\right\}$ и пусть $B_{k}(x)$ - семейство всех интервалов вида $H(I)$, где $H$ - гомотетия с центром в $x$ и $I \in \Delta_{k}(x)$. Обозначим

$$
N(x)=\left\{k \in \mathbb{N}: x \in \bigcup_{q=1}^{q_{k}} \widetilde{S}_{k, q}\right\}, \quad x \in \mathbb{G}^{2} .
$$

Теперь базис $B$ определим следующим образом:

$$
B(x)= \begin{cases}\bigcup_{k \in N(x)} B_{k}(x) & \text { при } x \in \varlimsup_{k \rightarrow \infty} \bigcup_{q=1}^{q_{k}} \widetilde{S}_{k, q}, \\ \mathbb{I}(x) & \text { при } x \notin \varlimsup_{k \rightarrow \infty} \bigcup_{q=1}^{q_{k}} \widetilde{S}_{k, q} .\end{cases}
$$

Нетрудно видеть, что $B$ является инвариантным относительно гомотетии и центрированньпм.

Положим

$$
f_{k}=\sum_{q=1}^{q_{k}} h_{k} \chi_{I_{k, q}}, \quad k \in \mathbb{N}
$$

и $f=\sum_{k=1}^{\infty} f_{k}$. При $k \in \mathbb{N}$ имеем

$$
\left\|f_{k}\right\|_{1}=h_{k}\left|\bigcup_{q=1}^{q_{k}} I_{k, q}\right|=h_{k} \sum_{q=1}^{q_{k}} \frac{\left|Q_{k, q}\right|}{4 h_{k}^{2}}=\frac{1}{4 h_{k}},
$$

откуда в силу (11) заключаем, что $f \in L\left(\mathbb{G}^{2}\right)$.

Очевидно, $B$ дифференцирует $\int f_{k}, k \in \mathbb{N}$, т.е. множество $A_{k}$, состоящие из всех точек $x$, для которых $D_{B}\left(\int f_{k}, x\right)=f_{k}(x)$, имеет полную меру в $\mathbb{R}^{2}$.

Обозначим

$$
A=\left(\bigcap_{k=1}^{\infty} A_{k} \cap \varlimsup_{k \rightarrow \infty} \bigcup_{q=1}^{q_{k}} \widetilde{S}_{k, q}\right) \backslash \varlimsup_{k \rightarrow \infty} R\left(I_{k, q}, h_{k}\right) .
$$


Нетрудно видеть, что

$$
\left|\bigcup_{q=1}^{q_{k}} R\left(I_{k, q}, h_{k}\right)\right| \leqslant 12 h_{k} \sum_{q=1}^{q_{k}}\left|I_{k, q}\right|=12 h_{k} \sum_{q=1}^{q_{k}} \frac{\left|Q_{k, q}\right|}{4 h_{k}^{2}}=\frac{3}{h_{k}} .
$$

Следовательно, в силу (11)

$$
\left|\varlimsup_{k \rightarrow \infty} \bigcup_{q=1}^{q_{k}} R\left(I_{k, q}, h_{k}\right)\right|=0
$$

откуда на основании (17) заключаем, что $A$ имеет полную меру в $\mathbb{G}^{2}$.

Пусть $x \in A$. Ясно, что найдется $k_{x} \in \mathbb{N}$, для которого

$$
x \notin \bigcup_{q=1}^{q_{k}} R\left(I_{k, q}, h_{k}\right) \quad \text { при } k>k_{x} .
$$

Очевидно, при $\operatorname{diam} I \rightarrow 0, I \in B(x)$

$$
\frac{1}{|I|} \int_{I} \sum_{k=1}^{k_{x}} f_{k} \rightarrow \sum_{k=1}^{k_{x}} f_{k}(x)=f(x)
$$

Рассмотрим произвольньй интервал $I \in B(x)$. Обозначим

$$
k(I)=\min \left\{k>k_{x}: I \cap \bigcup_{q=1}^{q_{k}} I_{k, q} \neq \varnothing\right\} .
$$

Пусть $q \in \overline{1, q_{k(I)}}$ такова, что $x$ принадлежит замыканию $Q_{k(I), q}$. Тогда, учитывая, что $S_{k(I), \nu}, \nu \in \overline{1, q_{k(I)}}$, попарно не пересекаются, на основании леммы 10 заключаем

$$
\frac{1}{|I|} \int_{I}\left(f_{k(I)}-h_{k} \chi_{I_{k(I), q}}\right) \leqslant 1
$$

Если $x \in S_{k(I), q}$ и $I \in B_{k(I)}(x)$, то, учитывая (18) и лемму 9, напишем

$$
\frac{h\left|I \cap I_{k(I), q}\right|}{|I|} \leqslant 4 .
$$

Если же $I \in B_{k}(x)$, где $k \neq k_{x}$, то, заметив $r\left(I_{k, q}\right)=2^{m_{k}-n_{k}}$, из определения $B_{k}(x)$ и из леммы 7 нетрудно видеть, что

$$
r(I) \in\left[\frac{2^{m_{k}-n_{k}}}{h_{k}}, h_{k} 2^{m_{k}-n_{k}}\right] .
$$

Следовательно, на основании (14) пишем

$$
r(I) \notin\left[\frac{2^{m_{k(I)}-n_{k(I)}}}{h_{k(I)}}, h_{k(I)} 2^{m_{k(I)}-n_{k(I)}}\right],
$$


откуда, учитьвая, что $x \notin R\left(I_{k(I), q}, h_{k}\right)$ (см. $\left.(18)\right)$, в силу леммы 8 заключаем

$$
\frac{h\left|I \cap I_{k(I), q}\right|}{|I|} \leqslant 1 \text {. }
$$

Из (20)-(22) следует, что

$$
\frac{1}{|I|} \int_{I} f_{k(I)} \leqslant 5 \text {. }
$$

Так как $x \in I$ и $x \notin R\left(I_{k(I), q}, h_{k}\right)$, то $\beta_{I} \geqslant \beta_{I_{k(I), q}}=1 / h_{k(I)} 2^{m_{k(I)}+1}$, откуда в силу (12) и (13)

$$
\beta_{I} \geqslant \frac{1}{2^{n_{k}}}=\alpha_{I_{k, q}} \quad \text { при } k>k(I) \quad \text { и } \quad q \in \overline{1, q_{k}} .
$$

Пусть $P_{k}, k \in \mathbb{N},-$ множество всех вершин интервалов $Q_{k, q}, q \in \overline{1, q_{k}}$. Тогда в силу (24) при $k>k(I)$ найдется интервал $J$ с вершинами, принадлежащими $P_{k}$, такой, что $I \subset J \subset 3 I$, откуда, учитьвая очевидное равенство

$$
\left|J \cap \bigcup_{q=1}^{q_{k}} I_{k, q}\right|=|J|\left|\bigcup_{q=1}^{q_{k}} I_{k, q}\right|
$$

напишем

$$
\left|I \cap \bigcup_{q=1}^{q_{k}} I_{k, q}\right| \leqslant 9|I|\left|\bigcup_{q=1}^{q_{k}} I_{k, q}\right|
$$

Следовательно, для каждой $k>k(I)$

$$
\frac{1}{|I|} \int_{I} f_{k}=\frac{1}{|I|} h_{k}\left|I \cap \bigcup_{q=1}^{q_{k}} I_{k, q}\right| \leqslant 9 h_{k}\left|\bigcup_{q=1}^{q_{k}} I_{k, q}\right|=9\left\|f_{k}\right\|_{1} .
$$

Стало быть,

$$
\frac{1}{|I|} \int_{I} \sum_{k>k(I)} f_{k} \leqslant \sum_{k>k(I)} 9\left\|f_{k}\right\|_{1} \leqslant 9\|f\|_{1}
$$

Очевидно также, что

$$
\int_{I} f_{k}=0 \quad \text { при } \quad k_{x}<k<k(I) .
$$

Из (19), (23), (25) и (26) легко заключаем

$$
\bar{D}_{B}(f, x) \leqslant f(x)+5+9\|f\|_{1} .
$$

Так как $x \in A$, найдутся последовательности $\{k(j)\}, k(j) \rightarrow \infty$, и $\{q(j)\}, 1 \leqslant q(j) \leqslant q_{k(j)}$, для которых $x \in S_{k(j), q(j)}$ при каждом $j \in \mathbb{N}$. В силу леммы 6 при каждом $j \in \mathbb{N}$ найдется интервал $R_{j} \in \Lambda\left(I_{k(j), q(j)}, h_{k(j)}\right)$, которьй содержит $x$. Легко видеть, что

$$
\begin{gathered}
\left\{R_{j}\right\} \subset B(x), \quad \frac{1}{\left|R_{j}\right|} \int_{R_{j}} f_{k(j)} \geqslant \frac{1}{\left|R_{j}\right|} \int_{R_{j}} h_{k(j)} \chi_{I_{k(j), q(j)}}=1, \\
\operatorname{diam} I \leqslant \operatorname{diam} Q_{k(j), q(j)}<\frac{1}{2^{n_{k(j)}-1}},
\end{gathered}
$$

откуда с учетом (19) легко видеть, что $\bar{D}_{B}(f, x) \geqslant f(x)+1$. Так как $A$ имеет полную меру в $\mathbb{G}^{2}$ и точка $x \in A$ была выбрана произвольно, учитьвая (27) заключаем справедливость теоремы. 


\section{СПИСОК ЦИТИРОВАННОЙ ЛИТЕРАТУРЫ}

[1] Гусман М. Дифференцирование интегралов в $\mathbb{R}^{n}$. М.: Мир, 1978.

[2] Saks S. Remarks on the differentiability of the Lebesgue indefinite integral // Fund. Math. 1934. V. 22. P. 257-261.

[3] Безикович A. C. On differentiation of Lebesgue double integrals // Fund. Math. 1935. V. 25. P. 209-216.

[4] Ward A. On the derivation of additive functions of intervals in $m$-dimensional space // Fund. Math. 1937. V. 28. P. 265-279.

[5] Сакс С. Теория интеграла. М.: ИЛ, 1949.

[6] de Guzmán M. Real Variable Methods in Fourier Analysis. V. 46. Amsterdam: North-Holland Math. Stud., 1981.

[7] Korenovskyy A., Lerner A., Stokolos A. On multidimensional F.!Riesz's "rising sun" lemma // E-print arXiv:math.CA/0308211v1 (22 August), 2003.

[8] Zerekidze T. Differentiation of integrals by bases of type $\Pi$ // Proc. A. Razmadze Math. Inst. 2003. V. 133. P. 119-130.

[9] Zerekidze T. On the equivalence and nonequivalence of some differential bases // Proc. A. Razmadze Math. Inst. 2003. V. 133. P. 166-169.

[10] Ониани Г. Г. О возможных значениях верхней и нижней производных // Матем. заметки. 1998. Т. 64. № 1. С. 107-114.

[11] Ониани Г. Г. Дифференцирование интегралов Лебега. Тбилиси: Изд-во Тбилисского ун-та, 1998.

[12] Busemann H., Feller W. Zur Differentiation der Lebesgueschen Integrale // Fund. Math. 1934. V. 22. P. 226-256.

[13] Oniani G. On strong maximal operators corresponding to different frames (II) // Georgian Math. J. 1999. V. 6. № 2. P. 149-168.

[14] Jessen B., Marcinkiewitcz J., Zygmund A. Note on the differentiability of multiple integrals // Fund. Math. 1935. V. 25. P. 217-234.

Кутаисский государственный университет им. А. Церетели, Грузия

Поступило

E-mail: tava73@mail.ru

20.01.2003 